\title{
Fatigue life assessment of a railway wheel material under HCF and VHCF conditions
}

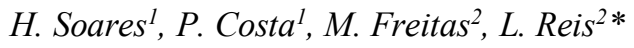 \\ ${ }^{1}$ Instituto Superior Técnico, Universidade de Lisboa, Av. Rovisco Pais, 1, 1049-001 Lisbon, Portugal \\ ${ }^{2}$ IDMEC, Instituto Superior Técnico, Universidade de Lisboa, Av. Rovisco Pais, 1, 1049-001 Lisbon, Portugal
}

\begin{abstract}
Fatigue damage and life assessment is still an issue and a challenge nowadays. Many different tests can be performed for the assessment of fatigue properties of any given material. In the present study a worn out railway wheel goes under uniaxial fatigue analyses for the high cycle and very high cycle fatigue regimes through the use of a conventional hydraulic machine and an ultrasonic fatigue test, both with a stress ratio of $\mathrm{R}=-1$. For every used specimen, a life cycle is obtained for the corresponding induced specimen and the data is afterwards plotted. All the fracture surfaces of all failed specimens were analysed. The study and initial application of multiaxial ultrasonic fatigue condition and the design of the corresponding specimen for evaluating the wheel material is also presented.
\end{abstract}

\section{Introduction}

The fatigue study is of the most importance for any system subjected to dynamic loadings being the most common and unpredictable type of failure. It has been shown that fatigue in metals may occur in any range of cycles, even beyond what was once considered the fatigue life limit [1].

To study the fatigue of a certain material several tests can be performed to relate the fatigue life for low cycle fatigue (LCF), high cycle fatigue (HCF) and very high cycle fatigue (VHCF). In any given range different specimens and displacements can be induced depending on the regime and type of load of interest, uniaxial or multiaxial. For LCF and HCF regimes, conventional hydraulic machines are employed which induce stress cycles at a low rate of around $50 \mathrm{~Hz}$, easy to apply and control. In VHCF regime, in the $10^{6}$ and $10^{9}$ cycle range, ultrasonic resonance methods are employed to induce stress cycles with a frequency of around $20 \mathrm{kHz}$. This type of tests allows to perform a huge number of cycles which it would be unpractical for a conventional machine.

Ultrasonic fatigue tests apply resonance principles for the achievement of high enough stress at a high frequency to an excited specimen. Mason was the first to achieve a working system in 1950 [2] with a frequency of work of $20 \mathrm{kHz}$, establishing the standard frequency of all methods created afterwards. Since then, many researchers have dedicated to the development of new variations of this type of test capable of applying different types of loadings, stress ratios and also in different ambient conditions, like those seen in $[3 ; 4]$.

The present study applies uniaxial stress, tensile and compression cycles, with a stress ratio of $\mathrm{R}=-1$ to a wornout railway wheel using a conventional hydraulic machine
Instron 8874 for $\mathrm{HCF}$, and an ultrasonic fatigue testing with a device built in the Instituto Superior Técnico at the IDMEC laboratories [5] for the study of fatigue life in the VHCF regime. All material samples were machined from the railway wheel rim itself. Soul components used for ultrasonic fatigue test are showed in figure 1.

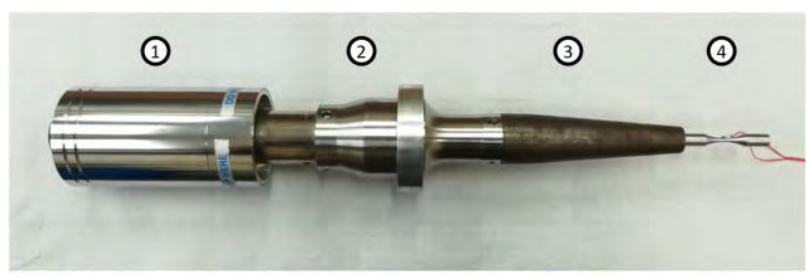

Fig. 1. Components to perform a uniaxial ultrasonic fatigue test: 1-Piezoelectric transducer; 2- Booster; 3-Horn; 4-Test Specimen.

The piezoelectric transducer transforms electric pulses in mechanical pulses and is responsible for the resonance excitement of all the attached components. The main purpose of the Booster and the horn is to amplify the displacement of the transmitted transducer pulses. The specimen has an hourglass shape for the creation of higher stresses. Thereby with the use of the booster, horn and the specimens shape it is possible to achieve high enough stresses with a low power and a high frequency of $20 \mathrm{kHz}$.

Many different studies of uniaxial ultrasonic fatigue were already made by several different authors, as it can be seen in $[6 ; 7]$.

A multiaxial ultrasonic fatigue test is also mentioned, built in the same facilities using the same piezoelectric machine [8] and some of the preliminary trials preformed.

\footnotetext{
* corresponding author: luis.g.reis@tecnico.ulisboa.pt
} 


\section{Methodology}

The material in study is a worn-out railway wheel that should follow the European norm EN_13262+A1-2009.

For both the HCF and VHCF tests Young Modulus is required for the specimen's creation and applied stress. Material density is also required in the ultrasonic fatigue test for the determination of resonance frequency. These material properties were obtained through a tensile stress test and by measuring the weight and volume of a certain part of the railway wheel rim.

\subsection{High cycle fatigue}

For any conventional HCF test a standard specimen is need it. The used geometry follows the standard ASTM E606 (2003) for axial fatigue tests. The machined geometry and the respective dimensions are presented in figure 2 .

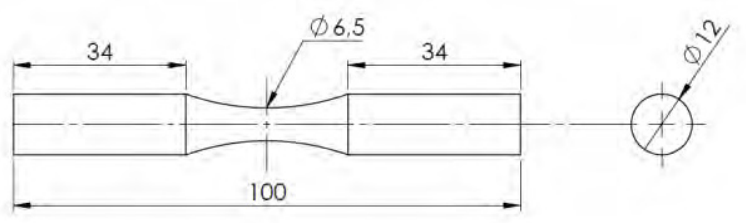

Fig. 2. HCF specimen geometry and dimensions following the standard ASTM E606 (2003) (mm).

As for the ultrasonic test specimen the conventional specimens are machined directly from the railway wheel rim.

All tests are executed through load control. A stress of interest for the study is chosen and with the Young Modulus and the measuring the radius of the smallest cross section the control force to be apply can be calculated. The specimen is then subjected to stress cycles until failure. If after 10E06 cycles the specimen didn't show any sign of failure it is withdrawn from the machine and higher stress is applied. When the specimen fractures the machine automatically stops, the cycles are registered and the specimen is withdrawn for the analysis of its fracture surface.

\subsection{Very high cycle fatigue}

Using the determined material proprieties, a preliminary geometry for the uniaxial ultrasonic test specimen was created through the base geometry and equations methodology found in [2].

After the initial calculations the geometry is applied in the finite element software Abaqus to be readjusted to a final design. The readjustment is made because the analytical method gives, according to finite element modal analysis, a significant lower frequency than 20 $\mathrm{kHz}$. Thereby a reduction of the cylindrical part of the specimen is made for increase of frequency until the resonance mode of interest is as close as possible to $20 \mathrm{kHz}$, maintaining a reasonable dimension for machining.
With the final geometry presented in figure 3 the specimens were machined delicately from the railway wheel rim.

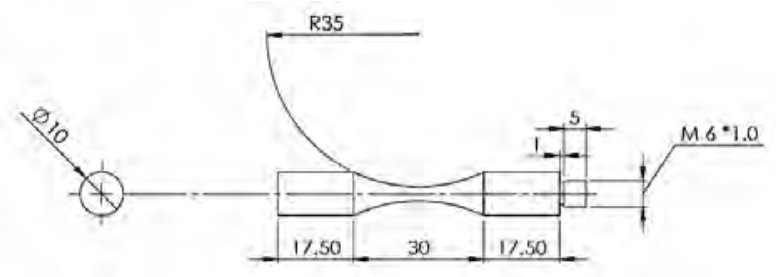

Fig. 3. Uniaxial ultrasonic fatigue test final specimen geometry and dimensions $(\mathrm{mm})$.

Before applying a constant stress cycle to any given specimen, a preliminary research of the created stresses is required. This was made through the usage of a strain gage to one of the machined specimens. For the first specimen in question several tests with different power control settings are applied. In each test the strains are measured, registered and worked through the use of the software MATLAB, thus obtaining the stress peak for each power setting applied. A trendline is then obtained linking the applied power with the resulting stresses.

\subsubsection{Strain measurements}

In order to measure the specimen's strain an HBM strain gage is applied to the smallest cross section where the strain should be the highest and consequently highest stress. Figure 4 shows a photograph of the strain gage applied. The strain gage is aligned with the induced displacement.

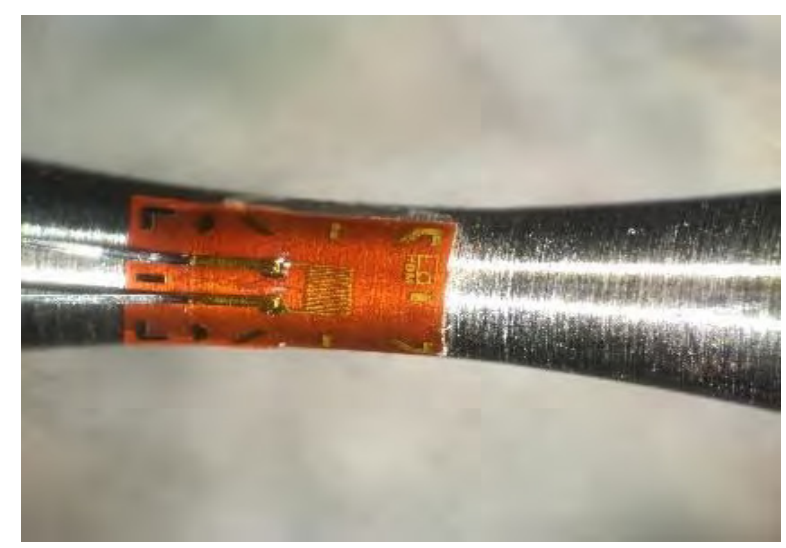

Fig. 4. HBM strain gage used and bounded in the smallest cross section.

The strain gage is connected to a data acquisition system for the recording of the strain data. Four power settings were applied and the corresponding strains were measured. The strains are then transformed to stress using the determined Young Modulus. Figure 5 and 6 show an interval of strain and derivative stress values respectively for a power setting of $10 \%$.

Through the use of the MATLAB software the maximum and minimum stresses are taken from a certain stable span and the average is considered to be the stress equivalent for the related power setting. Figure 7 shows 
the relation between stress and power setting and the corresponding trendline linear equation.

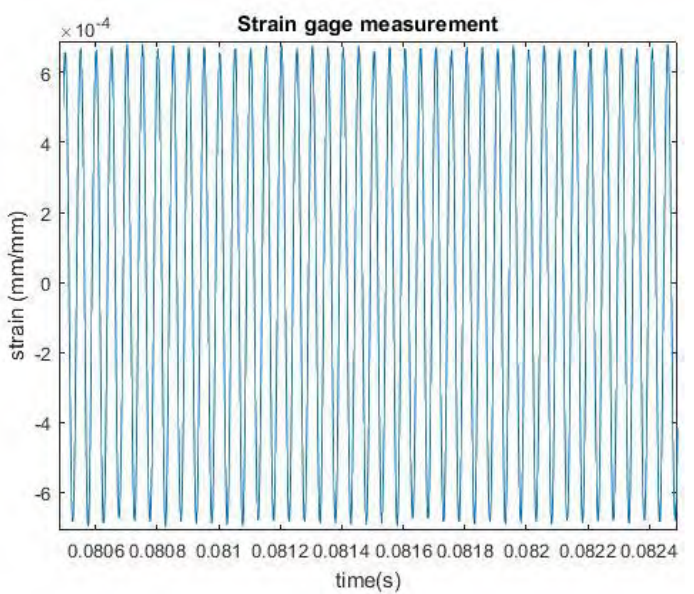

Fig. 5. Span of the measure strains for an applied power setting of $10 \%$.

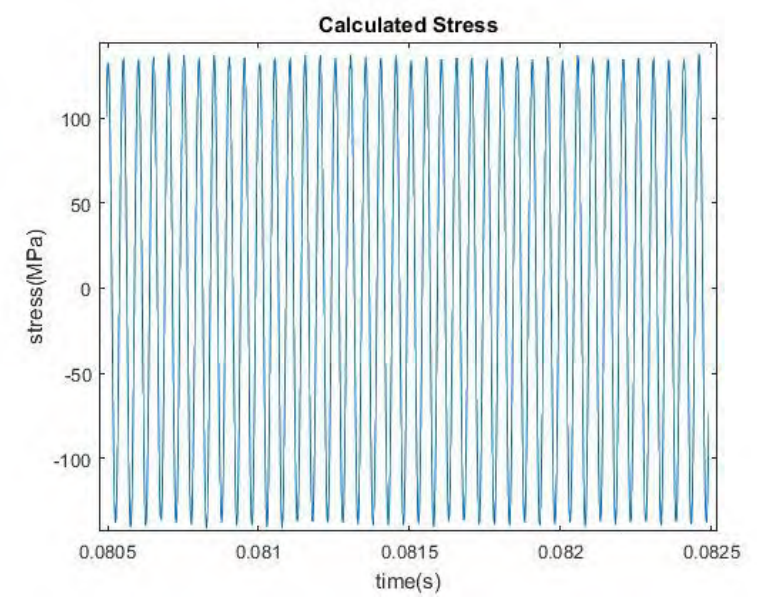

Fig. 6. Resulted stress from a span of measure strain for an applied power setting of $10 \%$.

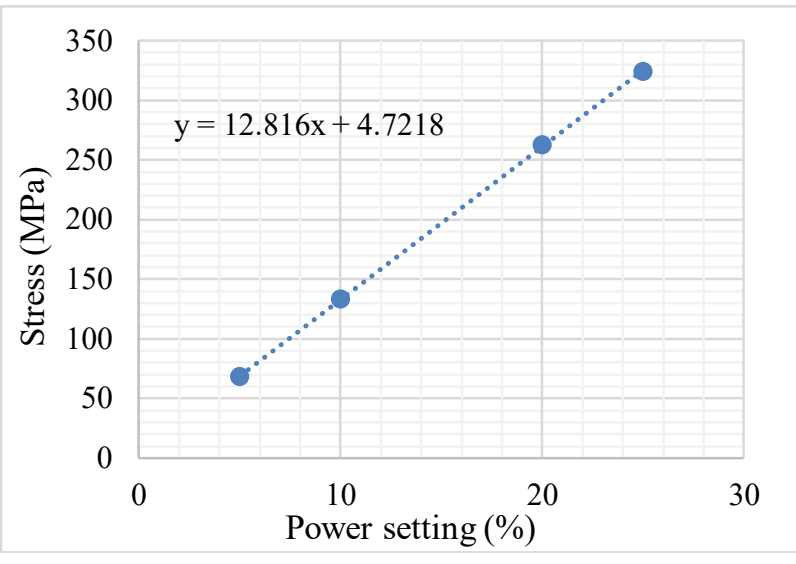

Fig. 7. Obtained relation between stress and power.

Using the obtained linear equation power/stress and knowing the supposed fatigue limit by HCF tests previously preform, ultrasonic fatigue tests were them carried out. The power settings used and the corresponding stresses of interest is presented in table 1 .
It is important to mention that the used piezoelectric transducer can only vary the power setting by half percent.

Table 1. Calculated stresses for the piezoelectric transducer power settings of interest

\begin{tabular}{|c|c|}
\hline Power (\%) & Average Stress $(\mathrm{MPa})$ \\
\hline 23.0 & 299.49 \\
\hline 23.5 & 305.90 \\
\hline 24.0 & 312.31 \\
\hline 24.5 & 318.71 \\
\hline 25.0 & 325.12 \\
\hline
\end{tabular}

\subsubsection{Stress cycles application}

Through the previously performed HCF tests, it was determined the stress at which the material won't break after being subjected to $10 \mathrm{E} 06$ cycles. This stress level corresponds to what it is called the fatigue limit. This and slightly lower stresses are the range of interest for the VHCF tests to be performed by the ultrasonic fatigue machine.

Several specimens are then tested below the threshold obtained by the HCF tests performed beforehand. Throughout any test a temperature control is applied due to the high heat generation during the test. A temperature limit is established and when reached the transducer is turned off temporarily until the specimen is cooled. For the measurement of the specimen's temperature all are previously painted in black. The higher heat generation comes from the region with higher induced stress where the damping effects are more influent. Figure 8 shows thermographic images of one specimen where it can be seen the heat generation on its smallest cross section.

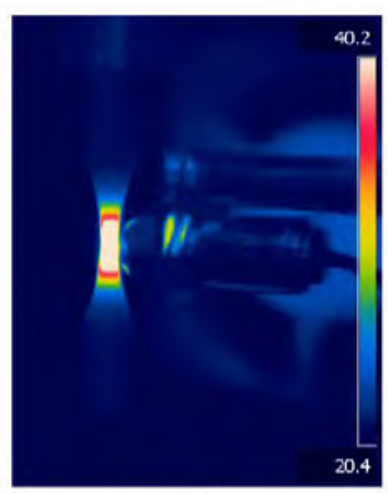

(A)

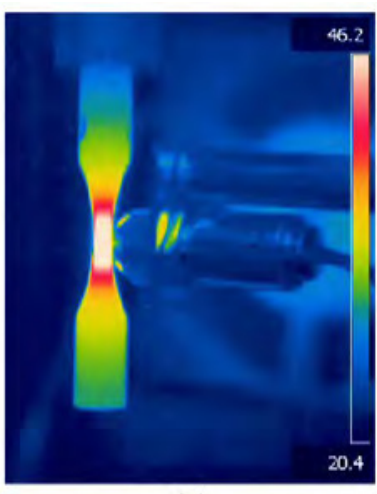

(B)
Fig. 8. Thermographic image of two tested ultrasonic uniaxial specimens: (A) beginning of a teste; (B) few minutes later.

For counting the applied cycles, a laser was used. The laser points directly to the free end of the specimen where the displacements are the highest, thus being able to count the cycles. It can also provide the frequency at which the test is currently working.

Opposite to the HCF conventional test the ultrasonic specimen does not completely break while in the ultrasonic fatigue machine. The test is finished when the resonance of the components has deviated from the range of work of the transducer. This happens when the fatigue damage to the specimen is big enough for it to lose 
integrity altering considerably his resonance frequency. Afterwards the specimen is withdrawn from the ultrasonic fatigue machine and a tensile test is made until the specimen's total failure.

\subsection{Multiaxial ultrasonic fatigue test}

The multiaxial ultrasonic test has the same methodology as the uniaxial. It only varies the horn and specimen geometry that allows to create the biaxial stress state of tension/compression and torsion.

The horn has a special geometry that partially transforms the longitudinal pulses created by the transducer to rotational pulses. Both types of displacement and rotation are then transmitted to the specimen. The specimen has also a special geometry that is able to be excited in two resonance modes simultaneously, the first longitudinal mode and the third rotational mode. With both of the referenced resonance modes it is possible to have one single area where both types of stresses achieved the highest value.

To create a final specimen geometry several finite element modal analysis are required until a final geometry with both resonance modes of interest have similar frequencies around $20 \mathrm{kHz}$. The final geometry and its dimensions are shown in figure 9.

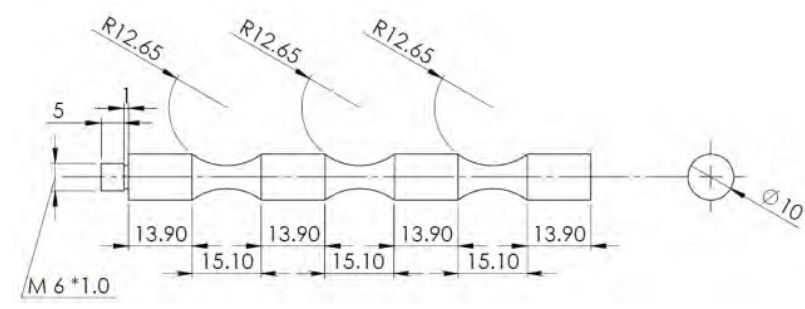

Fig. 9. Multiaxial ultrasonic fatigue test final specimen geometry and dimensions ( $\mathrm{mm}$ ).

After machining the multiaxial specimen, it is first validated through the generated temperature. The specimen is painted in black and excited while the temperature is observed by a thermal camera. The results have proven to be promising since two machined specimens have shown to have the higher heat generation on the intended location, as it can be seen in figure 10 .

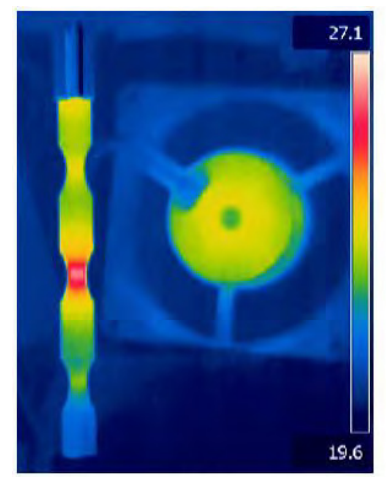

(A)

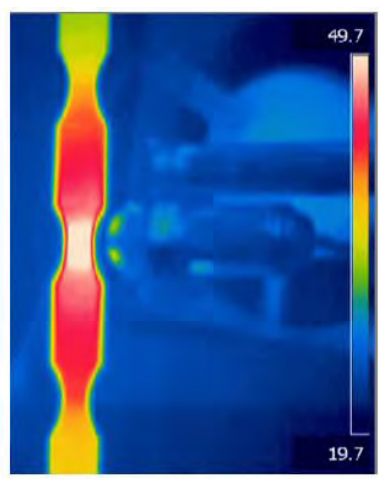

(B)
Fig. 10. Thermographic image of two tested ultrasonic multiaxial specimens at: (A) low power setting; (B) higher power setting.
The next step for the study is the introduction of a strain gage with three measuring gages thus providing the ability to obtain the shear stress created by the induced torsion.

\section{Results and Discussion}

\subsection{High cycle fatigue}

Studying the results, we can confirm that the material reaches $10 \mathrm{E} 06$ cycles around $325 \mathrm{MPa}$. Thereby it should be applied to the ultrasonic fatigue specimen stresses below this value.

All fracture surfaces of failed specimens showed to have a zero degree as it is expected for a conventional tension/compression fatigue test. Through the analysis of the fracture surface of all failed specimens the fatigue propagation initiated at the surface. Figure 11 shows the fracture angle and the initial site of propagation.
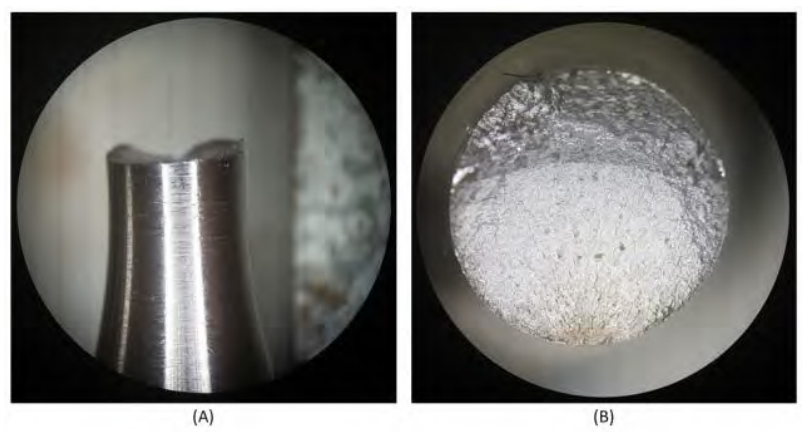

Fig. 11. (A) Fracture angle; (B) fracture surface.

\subsection{Very high cycle fatigue}

The ultrasonic fatigue specimens showed as with the conventional test results to have the fracture surface with a zero-degree angle of failure. The fracture surface also shows to have initiated at the specimen's surface. Figure 12 shows the fracture angle and the initial site of propagation.

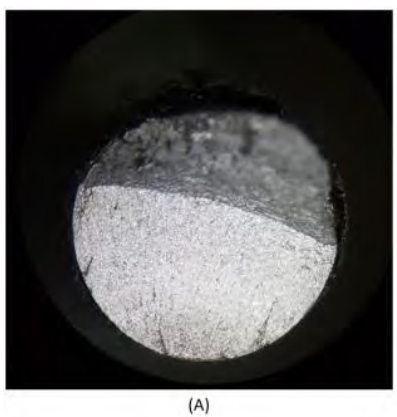

(A)

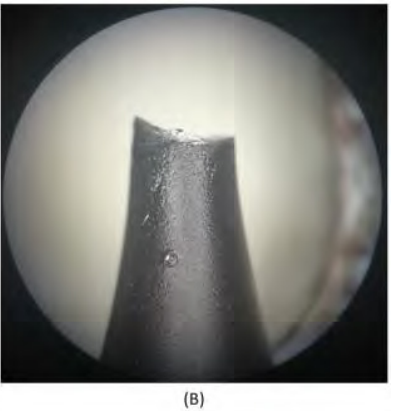

(B)
Fig. 12. (A) Fracture surface; (B) fracture angel. 


\subsection{S-N curve of HCF and VHCF results}

Putting all the results of both preformed tests a stress life cycle graph is plotted, as it can be seen in Figure 13.

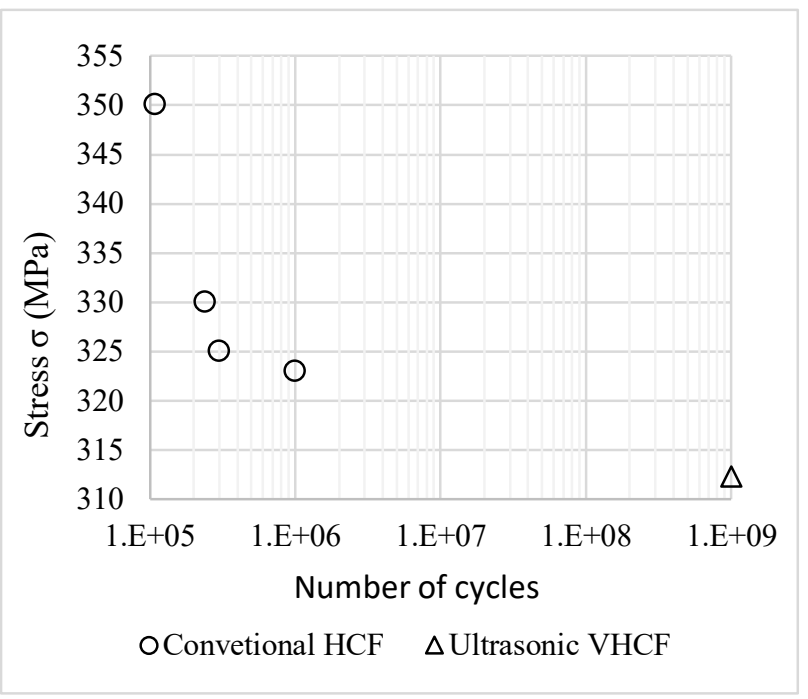

Fig. 13. Stress life results of conventional HCF and ultrasonic VHCF tests.

Observing the results, it is not possible with the points so far obtained to fully characterize the material behaviour in the VHCF regime. The HCF results show with clarity the increase of material's life with stress reduction until the VHCF regime. In the VHCF regime, a stress reduction shows to have a much more significant impact on material life.

\section{Final Remarks}

Comparing the obtained fracture surfaces between the performed tests there are no obvious differences. For the two studied fatigue regimes fracture initiation site showed to be in the specimen's surface. The fracture surface marks, angle of failure and even the shape seem to be similar at eye sight and through a microscope, as it can be compared between figures 11 and 12. A detailed and more powerful magnification of the surface would be required to completely assess the differences between the fracture morphologies of both tests.

More tests are being done in order to fully characterize the material behaviour in the VHCF regime.

\section{References}

1. Bathias, C., Fract Engng Mater Struct, 22, 559-565 (1999).

2. Bathias, C., \& Paris, P. C. (2005). Gigacycle Fatigue in Mechanical Practice (1st ed.). New York: Marcel Dekker.

3. Bathias, C., International Journal of Fatigue, 28, 1438-1445 (2006).
4. Xue, H. Q., Tao, H., Montembault, F., Wang, Q., \& Bathias, C., 29, 2085-2093 (2007).

5. Freitas, M., Reis, L., Anes, V., Montalvão, D., Ribeiro, A. M., \& Fontes, M. Anales de Mecánica de la Fractura, 1, 335-340 (2011).

6. Mayer, H., International Journal of Fatigue, 28, 14461455 (2006).

7. Marines, I., Dominguez, G., Baudry, G., Vittori, J. F., Rathery, S., Doucet, J. P., \& Bathias, C., International Journal of Fatigue, 25, 1037-1046 (2003).

8. Vieira, M., Freitas, M. d., Reis, L., Ribeiro, A. M., \& Fonte, M. d., 37, 131-137 (2016). 